\title{
Medicinal plants sold for treatment of bacterial and parasitic diseases in humans in Maputo city markets, Mozambique
}

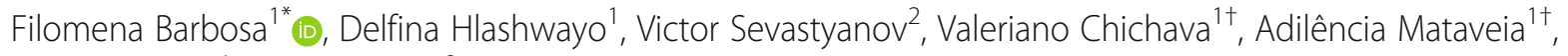
Ernesto Boane ${ }^{1}$ and Aida Cala ${ }^{3}$

\begin{abstract}
Background: In Mozambique, bacterial and parasitic diseases contribute to a high burden of mortality and morbidity. These infectious diseases are treated with antibiotics, antihelmintic or antiparasitic drugs. However, misuse of these has been affecting the potential to treat ailments. It has been reported that many people from Maputo city and province apart from the existing contemporary medicine, still use medicinal plants for treatment of diseases due to traditional heritage and beliefs. It is, therefore, important to register this knowledge in order to use it for future pharmacological studies. This study aimed to identify the medicinal plants sold in Xipamanine, Xiquelene and Mazambane markets for treatment of bacterial and parasitic diseases.
\end{abstract}

Methods: An ethnobotanical survey, using interviews, was conducted to the main vendors of the markets. Data about the plant name, part used, mode of preparation and administration route were collected.

Results: A total of 64 medicinal plants belonging to 32 families were listed as sold for treatment of bacterial and parasitic diseases in the three markets. Terminalia sericea, Elephantorrhiza elephantina, Tiliacora funifera and Hypoxis hemerocallidea were the most cited plants. Roots were the most often sold suggesting it is the most used part. We also found out that medicinal plants trade is still common in Maputo markets. This suggests that people still use plant-based herbal medicines for their basic health care.

Conclusions: Several medicinal plants were sold in Maputo city's markets for treatment of bacterial and parasitic diseases, with more emphasis on diarrhea and helminthiases. These plants were commonly bought by local residents and play an important role in the subsistence of vendors. Pharmacological studies are needed in order to isolate the plants active principles and understand their mechanism of action, so that new drugs can be developed.

Keywords: Medicinal plants, markets, Maputo, Mozambique

\section{Background}

Mozambique is a developing country in the Eastern region of Sub-Saharan Africa. According to the World Health Organization (WHO), almost $80 \%$ of the population in developing countries depends mainly on traditional medicine for treatment of diseases [1]. This scenario is also observed in Mozambique, where the majority of population (70\%) lives in the rural areas [2].

\footnotetext{
*Correspondence: filomena.barbosa97@gmail.com

†Valeriano Chichava and Adilência Mataveia contributed equally to this work

${ }^{1}$ Departamento de Ciências Biológicas, Faculdade de Ciências, Universidade

Eduardo Mondlane, Avenida Julius Nyerere nr 3453, Campus Principal,

Maputo, Mozambique

Full list of author information is available at the end of the article
}

The national healthcare system cannot cover the entire population and some medicines are expensive. Therefore, the population adheres to traditional medicine services $[3,4]$.

More than 5500 plant species are available in Mozambique and almost 10\% are used in the traditional medicine [5]. The trade of medicinal plants in Maputo is known since 1980's when only 10 traders were found in Xipamanine market [6]. Nowadays, 192 vendors are registered in the Association of Traditional Remedies Vendors from Mozambique (AVEMETRAMO: Associação dos Vendedores de Medicamentos Tradicionais) (unpublished data from 2017). These vendors sell 
medicinal plants and other non-herbal remedies in the main three medicinal plant markets, namely: Xipamanine, Xiquelene and Mazambane (also known as Adelino) [6].

Bacterial and parasitic diseases have a high burden in Mozambique [7]. The most common bacterial infections cause tuberculosis and diarrhea [7]. Diarrhea, a common symptom of intestinal infection by bacteria and other microorganisms, is among the main causes of morbidity and mortality in children under 5 years old in the country [8]. On the other hand, intestinal parasites, including helminthes and protozoa constitute a major cause of morbidity and mortality in the country [9], where about 11.730.145 children require preventive chemotherapy for soil transmitted helminthiases [10].

Many medicinal plant species have been cited by the vendors of the three markets in a previous ethnobotanical study [6]. With the upcoming antibiotic resistance of many drugs, there is a need for a continuous register of the sold plants, in order to conduct pharmacological studies aiming to develop new and effective drugs. It is likewise important to understand whether medicinal plants are still traded in these markets.

In addition to the advantages described above, there is a need to record existing ethnobotanical knowledge in the markets, to avoid erosion of knowledge. The identification of medicinal plants traded in the markets also gives an indication about the conservation status of species under trade. This type of study also helps to inform the current adherence of the population to the traditional medicine services.

The aim of this study was to identify the medicinal plants sold for treatment of bacterial and parasitic diseases in the three medicinal plant markets in Maputo city, as well as to register data regarding plant names, used parts, mode of preparation and administration routes.

\section{Methods}

\section{Study site}

The study was conducted in the three main medicinal plants trade markets in Maputo city, namely: Xipamanine, Xiquelene and Mazambane.

The city administratively constitutes a Municipality with an elected government and also has the status of Province since 1980. This municipality is divided into seven Municipal districts, namely KaMpfumo, Nlhamakulu, KaMaxakeni, KaMubukwana, KaMavota, KaTembe and KaNyaka. These districts are subdivided into neighborhoods for a total of 63 . Territorially, it is the smallest province of the country of $346.77 \mathrm{Km}^{2}$. It currently has 1,101,170.3 inhabitants according to 2017 census, with one of the highest population densities of the country, with 670.6 inhabitants / $\mathrm{Km}^{2}$ [11].
The high population density in less urbanized districts combined with poor housing conditions and lack of basic infrastructure make these districts or a part of their neighborhoods the most vulnerable to infectious diseases [12], which are still treated through medicinal plants by many social groups [6].

\section{Ethical compliance}

No ethical approval was obtained because there was no mechanism in place to obtain such approval in the country for studies that do not involve a prospective assessment, laboratory animals and invasive species. Nevertheless, the study complied with the International Society of Ethnobiology (ISE) Code of Ethics [13] and the local legislation on traditional knowledge [14]. The project was also authorized by the Department of Biological Sciences of Eduardo Mondlane University. Approval to conduct research in the markets was obtained from Maputo City Council (Conselho Municipal de Maputo) (credential number 105/2018). Prior to data collection, the project was explained to the markets managers in order to tell the objectives of research and to guarantee the safety of indigenous knowledge. The managers gave support in introducing investigators to the "leaders" of the vendors. The aim of the study was clearly explained and all interviewed vendors were asked for their prior oral consent.

\section{Collection of ethnobotanical information}

Ethnobotanical data was collected between January and February 2019 for two weeks. The research team went to Xipamanine market where the data was collected in the first week because it has the majority of medicinal plant vendors. Mazambane and Xiquelene markets were visited in the second week. Snow ball sampling was used. In Xipamanine and Xiquelene markets, the managers referred us to one of the "leaders" (so called because they have more experience in selling medicinal plants), which forwarded us to other "leaders". This procedure was repeated until we reached the last leader vendor. There are many vendors in the markets, but few have authorization to speak, possibly due to few experience in trade and because they are still learning from the leaders. In Mazambane market, we interviewed the only two vendors of medicinal plants. Using semi-structured interviews, vendor's socio-demographical information and data related to medicinal use of plants and other remedies were captured. Interviews were made in local language (Xitsonga/Xichangana) or Portuguese according to the informant's preference. In total, 15 interviews were carried out in the three markets. Personal information included: name, gender, age, place of birth, nationality, education levels, how they acquired knowledge about medicinal plants, if they had training on adequate 
storage of medicinal plants and years of experience on trade. Data was collected regarding plants traded, prices, place of harvest, local names, disease treated, preparation method and administration routes.

\section{Plant collection and identification}

Plant species sold or part of them were registered in local language and identified locally by the botanical technicians and compared with voucher from the University's herbarium (LMU), Maputo - Mozambique. Unknown plants specimen and/or parts sold were purchased and vouchers were made from those. These vouchers were deposited at the Eduardo Mondlane University's LMU Herbarium, Maputo - Mozambique. Plant identification was done through vernacular name in Xitsonga/Xichangana [15] and through purchased plant materials by botanical technicians from Eduardo Mondlane University, namely: Mr. Ernesto Boane, Mr. Ernesto Nacamo and Eng. Aurélio Bechel. The plant names were confirmed through http://www.theplantlist. org at June 5, 2019. Botanical families followed Angiosperm Phylogeny Group (APG) IV system [16].

\section{Data analysis}

Quantitative analysis of ethnobotanical data was done by calculating relative frequency of citation (RFC) and use value (UV) for all identified plants. Fidelity level (FL) was calculated for the most cited plants.

RFC was calculated by equation $[\mathrm{RFC}=\mathrm{FC} / \mathrm{N}]$ where $\mathrm{FC}$ is the frequency of citation of the mentioned species and $\mathrm{N}$ is the number of the interviewees [17]. The UV was computed by the number of uses mentioned by each informant for a specie (Ui) divided by the number of interviewees $(\mathrm{N})[\mathrm{UV}=\mathrm{Ui} / \mathrm{N}]$ [18]. Fidelity level was calculated by the number of informants that reported the use of a specie to treat a particular disease $(\mathrm{Np})$ divided by the number of informants that cited the use of the specie for any finality (Ns) [FL = Np/Ns] [19].

\section{Results}

\section{Sociodemographic information}

The majority of vendors were from Xipamanine and Xiquelene markets. Mazambane market had more sellers but many abandoned the place due to the construction work done in the market, which reduced the available space and subsequently led to the payment of a fee for the sale. On the other hand some vendors died. Only one vendor was female. Most of trader had the aged between 50 and 59 years and attended between 6th and 9th grade. They learned about medicinal plants from their families (mother, father, grandparents, sisters and brothers) and only one stated that learned from spirits that taught him through dreams. All vendors were Mozambicans. Almost half $(53.3 \%, n=8)$ of the vendors attended a training in adequate storage conducted by Direcção Nacional de Medicina Tradicional e Alternativa, the former Instituto de Medicina Tradicional from the Minister of Health. Most of the vendors had between 10 and 34 years of experience in selling medicinal plants. This suggests that they have extensive knowledge in medicinal plants. Detailed socio-demographic data is on Table 1.

\section{Medicinal plants sold in the markets}

A total of 64 plants were listed as sold for treatment of bacterial and parasitic diseases in the three markets. Fourteen plants, although mentioned by interviewers, were not identified because they were not available during data collection.

Table 1 Sociodemographic information of the vendors

\begin{tabular}{|c|c|}
\hline & N (\%) \\
\hline \multicolumn{2}{|l|}{ Market } \\
\hline Xipamanine & $6(40.0 \%)$ \\
\hline Xiquelene & $7(46.7 \%)$ \\
\hline Mazambane & $2(13.3 \%)$ \\
\hline \multicolumn{2}{|l|}{ Gender } \\
\hline Male & $14(93.3 \%)$ \\
\hline Female & $1(6.7 \%)$ \\
\hline \multicolumn{2}{|l|}{ Age range } \\
\hline 29-39 & $5(33.3 \%)$ \\
\hline $40-49$ & $4(26.7 \%)$ \\
\hline $50-59$ & $6(40.0 \%)$ \\
\hline \multicolumn{2}{|l|}{ Place of birth } \\
\hline Gaza & $9(60.0 \%)$ \\
\hline Inhambane & $2(13.3 \%)$ \\
\hline Maputo & $4(26.7 \%)$ \\
\hline \multicolumn{2}{|l|}{ Schooling } \\
\hline No schooling & $1(6.7 \%)$ \\
\hline $1-5$ th grade & $4(26.7 \%)$ \\
\hline 6-9th grade & $6(40.0 \%)$ \\
\hline 10-12th grade & $4(26.7 \%)$ \\
\hline \multicolumn{2}{|c|}{ Learning about medicinal plants } \\
\hline Family & $12(80.0 \%)$ \\
\hline Self/ spirits & $1(6.7 \%)$ \\
\hline Other vendors & $2(13.3 \%)$ \\
\hline \multicolumn{2}{|c|}{ Training on adequate storage } \\
\hline Yes & $8(53.3 \%)$ \\
\hline No & $7(46.7 \%)$ \\
\hline \multicolumn{2}{|c|}{ Years of experience in trading medicinal plants } \\
\hline $10-34$ & $12(80.0 \%)$ \\
\hline $35-50$ & $3(20.0 \%)$ \\
\hline
\end{tabular}




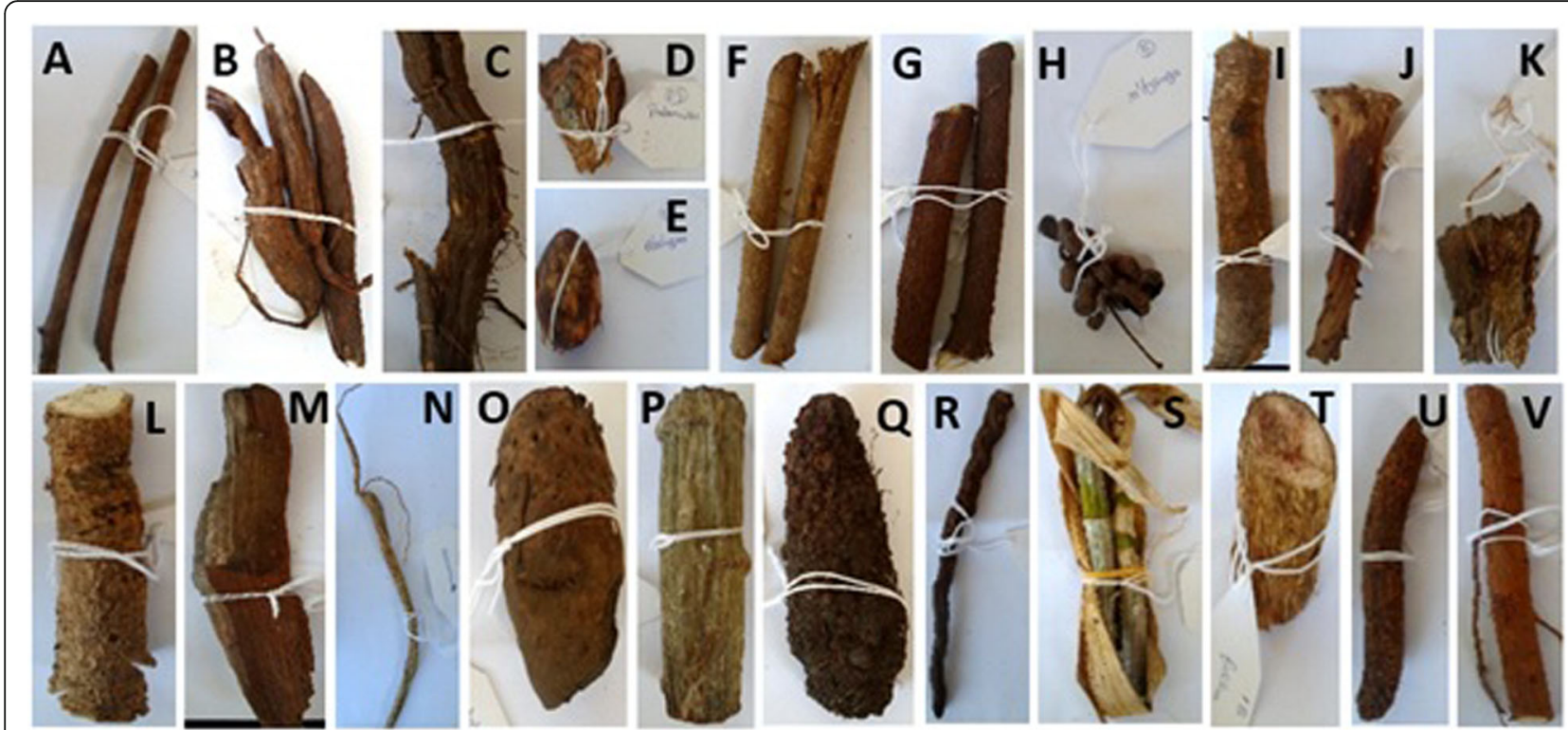

Fig. 1 Photographs of some medicinal plant parts sold in Xipamanine, Xiquelene and Mazambane markets. A Margaritaria discoidea roots B Elephantorrhiza elephantina roots C Tiliacora funifera root $\mathbf{D}$ Adenia gummifera root $\mathbf{E}$ Gladiolus sp. bulb $\mathbf{F}$ Terminalia sericea root $\mathbf{G}$ Gymnosporia heterophylla root $\mathbf{H}$ Dichrostachys cinerea fruit I Gymnanthemum coloratum root $\mathbf{J}$ Aloe marlothii dry leaf piece $\mathbf{K}$ Kedrostis sp. root $\mathbf{L}$ Strychnos spinosa root $\mathbf{M}$ Spirostachys africana root $\mathbf{N}$ Cucumis africanus root $\mathbf{O}$ Hypoxis hemerocallidea corm $\mathbf{P}$ Adenia gummifera root $\mathbf{Q}$ Hydnora abyssinica rhizome $\mathbf{R}$ Senna occidentalis root $\mathbf{S}$ Ansellia africana stem $\mathbf{T}$ Mucuna coriacea root $\mathbf{U}$ Ochna natalitia root $\mathbf{V}$ Garcinia livingstonei root

Figure 1 shows some of the medicinal plants sold in the markets. The most cited plants were Terminalia sericea, Elephantorrhiza elephantina, Tiliacora funifera and Hypoxis hemerocallidea with RFCs of $0.87,0.80,0.60$ and 0.53 , respectively. The plants belonged to 32 families, and the most frequent was Fabaceae with 6 species (Fig. 2). Some plants had more than one local name (e.g. Senna occidentalis, locally known as "Nhokane", "Ndlha nhoka", "Ndlha nhokane", "Nhokane utsongo" and "Nhokane uculo"), that is related to the disease treated. "Nhokane", meaning "roundworm", is mainly used for the treatment of helminthiases. Hydnora abissinica, specie cited to treat UTI, helminthiases and internal wounds, was first recorded in southern Mozambique in a previous study [20]. It is well known by traders and traditional healers, therefore widely used within traditional medicine in southern Africa. This plant is rarely found by botanicals [20]. Phytochemical studies showed high tannin concentration in rhizomes which imparts a strong astringency and this may explain its efficacy in treating ailments of the digestive tract [21].

Fourteen plant materials could not be determined to family and species level in this study because these plant species were not available in the markets during the study period. It is more so important that in view of their ethnopharmacological importance these plant species deserve future attention for determination purpose.

Information on plant phytochemical studies and biological assays allows to know if their traditional use is validated. This information will be useful for defining subsequent studies of these plants.

Table 2 summarizes the ethnobotanical information collected in the markets regarding medicinal plant sold for treatment of bacterial and parasitic diseases. Roots were the most often sold and used parts (75\%) (Fig. 3). The most cited diseases were helminthiases (28\%) and diarrhea (18\%) (Table 3). Decoction was the most common preparation method (74\%) (Fig. 4), and oral administration route was the most common (79\%), followed by topical (17\%) and anal administration (4\%). FL calculation for the most sold species and diseases is available on Table 4.

The vendors did not have concrete information about the time period in which plant-based preparations could be stored. Some informants said that the decoctions should be consumed until boiled for the third time. Other vendors said that the decoctions should be consumed until they stop having a bitter taste. Many reported that decoctions can be stored for 2 to 6 days, while some stated that they can be stored for 1 month if kept on the fridge. This is important because it contributes to the effectiveness of the remedies and can lead to microbial contamination if the remedy is poorly stored or kept for extended periods.

Most plants are prescribed and traded as mixtures previously cut and prepared by the vendors (Fig. 5). Aloe marlothii, Celosia sp., Cucumis africanus, Elephantorrhiza elephantina, Hypoxis hemerocallidea, Kedrostis sp., 


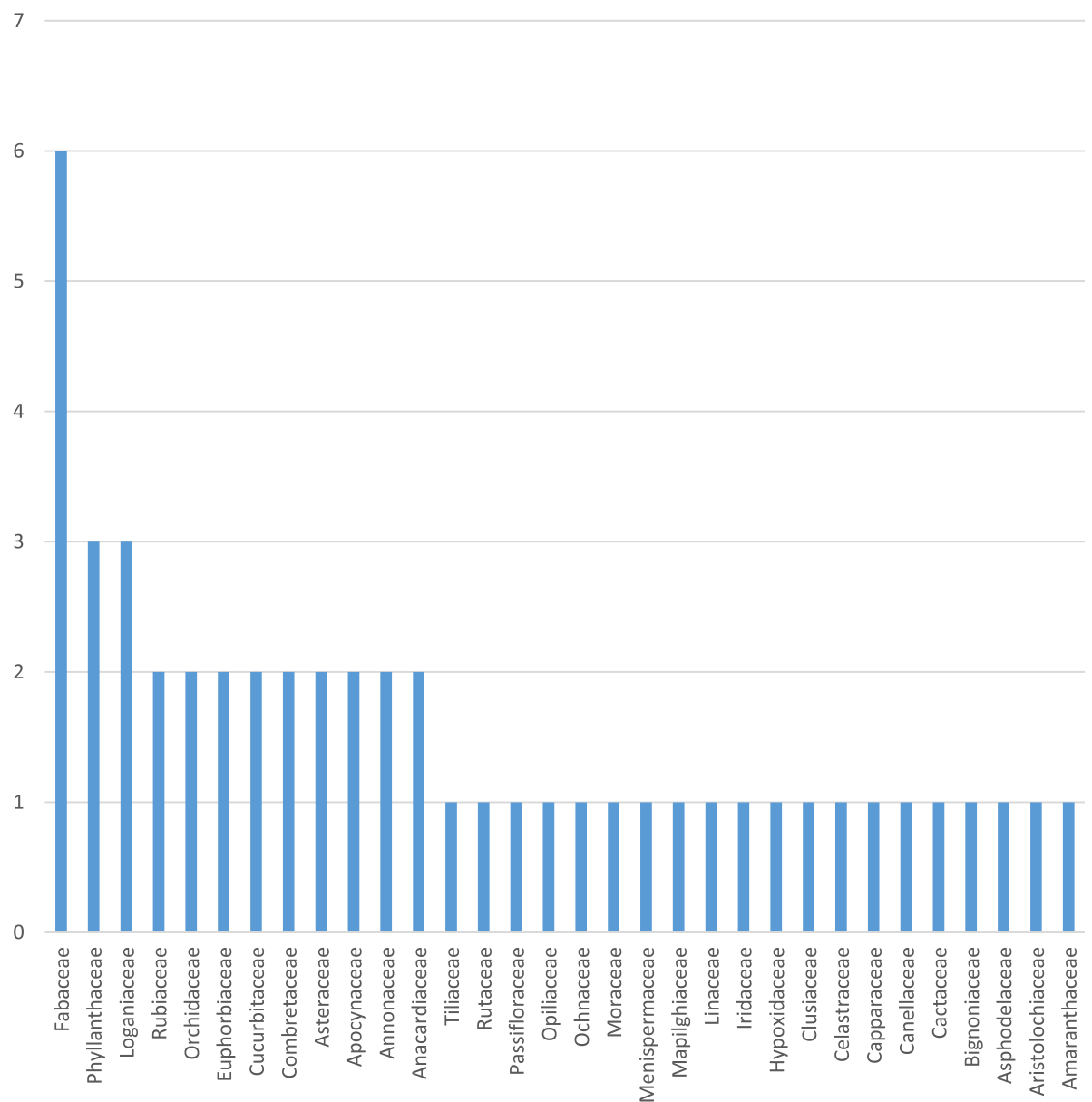

Fig. 2 Frequency of botanical families and number of species

Kigelia africana, Opuntia ficus-indica, Senna occidentalis, Strychnos henningsii, Terminalia sericea, Warburgia salutaris, "Lilhatana", "Nhanho", "Nicungo" and "Tsatsalane" were the only plants that were traded separately by some informants, although other informants sell these plants in mixtures.

When we asked the vendors about the price of their products, many reported that it depends on the consumer's buying capacity, i.e. if the consumer does not have financial resources, they will be charged a reduced price. Vendors affirmed that they are working to heal people and for this reason money does not matter much. Medicinal plant prices were very low and range from 10 MZN (0.16 USD) to $150 \mathrm{MZN}$ (2.43 USD). The average prices range from $10 \mathrm{MZN}$ to $50 \mathrm{MZN}$ (0.81 USD). The two highest priced plants are Warburgia salutaris, whose $15 \mathrm{~cm}$ piece of stem bark costed 100 MZN (1.62 USD) and Mangifera indica root, which was $150 \mathrm{MZN}$ (2.43 USD). An herbal mix used to treat cough had the highest price of $250 \mathrm{MZN}$ (4.05 USD). The mixture contained cut roots and leaves of the following plants: Annona senegalensis, Margaritaria discoidea, Grewia sulcata, Ansellia africana, Elephantorrhiza elephantina and "Chepa" (unidentified specie).

\section{Practices associated with the consumption of the remedies}

Some unusual practices are associated with remedies consumption, for example, W. salutaris stem bark decoction has to be dunk at hot-to-warm temperature on a cold day and at room temperature in a hot day for cough treatment. For helminthiases treatment, Cucumis africanus root decoction has to be drunk only when the sky is clear. These and other practices are common in traditional African medicine and sometimes refer to spiritual, empirical and traditional matters.

It was also noted that traditional medicine in Mozambique is not dissociated nor against conventional medicine, since many vendors advise the consumers to seek for medical services if the disease does not heal through medicinal plants.

Some informants cited other remedies that occur in the city and nearby places but are not sold. Burned snail shell (Achatina fulica) and Ricinus communis seed oil 
Table 2 Medicinal plants sold for treatment of bacterial and parasitic diseases in Xipamanine, Xiquelene and Mazambane markets, Mozambique

\begin{tabular}{|c|c|c|c|c|c|c|c|c|}
\hline $\begin{array}{l}\text { Scientific name / } \\
\text { voucher no }\end{array}$ & Family & Local name & $\begin{array}{l}\text { Parts } \\
\text { used }\end{array}$ & Ailment treated & Preparation & Administration & RFC & UV \\
\hline $\begin{array}{l}\text { Abrus precatorius } \\
\text { L. } 4336\end{array}$ & Fabaceae & Sissana & Root & Helminthiases & Decoction & Oral & 0.07 & 0.07 \\
\hline $\begin{array}{l}\text { Acanthospermum } \\
\text { hispidium DC. } \\
2624\end{array}$ & Asteraceae & Chinamane & Leaf & Wounds & Cold infusion & Topical & 0.07 & 0.07 \\
\hline $\begin{array}{l}\text { Acridocarpus } \\
\text { natalitius A.Juss. } \\
\text { Z445 }\end{array}$ & Mapilghiaceae & Mabope & Root & $\begin{array}{l}\text { Diarrhea (bloody or not), } \\
\text { haemorrhoids }\end{array}$ & Decoction & Oral & 0.13 & 0.13 \\
\hline $\begin{array}{l}\text { Adenia gummifera } \\
\text { (Harv.) Harms } \\
\text { MxiqDFB09 }\end{array}$ & Passifloraceae & Pindevemmushay & Root & $\begin{array}{l}\text { Internal wounds, } \\
\text { helminthiases, } \\
\text { tuberculosis }\end{array}$ & Decoction & Oral & 0.27 & 0.20 \\
\hline \multirow[t]{3}{*}{$\begin{array}{l}\text { Aloe marlothii A. } \\
\text { Berger JK7331 }\end{array}$} & \multirow[t]{3}{*}{ Asphodelaceae } & \multirow[t]{3}{*}{ Aloe vera, Mangane } & \multirow[t]{3}{*}{ Leaf } & Wounds & $\begin{array}{l}\text { Burn and apply the } \\
\text { hot sap or powder }\end{array}$ & $\begin{array}{l}\text { Topical (cover } \\
\text { or wash) }\end{array}$ & \multirow[t]{3}{*}{0.40} & \multirow[t]{3}{*}{0.27} \\
\hline & & & & Diarrhea, UTI & Decoction & Oral & & \\
\hline & & & & Cough & Mix with honey & & & \\
\hline \multirow{2}{*}{$\begin{array}{l}\text { Annona } \\
\text { senegalensis Pers. } \\
1644\end{array}$} & \multirow[t]{2}{*}{ Annonaceae } & \multirow[t]{2}{*}{ Rompfha } & \multirow[t]{2}{*}{ Root } & Wounds & Cold infusion & Topical -wash & \multirow[t]{2}{*}{0.13} & \multirow[t]{2}{*}{0.13} \\
\hline & & & & Cough & Decoction & Oral & & \\
\hline $\begin{array}{l}\text { Ansellia africana } \\
\text { Lindl. NM262 }\end{array}$ & Orchidaceae & $\begin{array}{l}\text { Phakama la hlanga } \\
\text { / Phakama }\end{array}$ & $\begin{array}{l}\text { Stem, } \\
\text { leaves }\end{array}$ & Cough, tuberculosis & Decoction & Oral & 0.20 & 0.13 \\
\hline Ansellia sp. K8132 & Orchidaceae & Phakama lankulho & Stem & Cough & Decoction & Oral & 0.07 & 0.07 \\
\hline $\begin{array}{l}\text { Artabotrys } \\
\text { brachypetalus } \\
\text { Benth. } 8198\end{array}$ & Annonaceae & N'tita & Root & Helminthiases & Decoction & Oral & 0.13 & 0.07 \\
\hline \multirow{2}{*}{$\begin{array}{l}\text { Bridelia cathartica } \\
\text { Bertol. K8084 }\end{array}$} & \multirow[t]{2}{*}{ Phyllanthaceae } & \multirow[t]{2}{*}{ Thlathlangati } & Root & Helminthiases & \multirow[t]{2}{*}{ Decoction } & \multirow[t]{2}{*}{ Oral } & \multirow[t]{2}{*}{0.13} & \multirow[t]{2}{*}{0.13} \\
\hline & & & Leaf & UTI & & & & \\
\hline $\begin{array}{l}\text { Catunaregam } \\
\text { spinosa (Thunb.) } \\
\text { Tirveng. KGD9651 }\end{array}$ & Rubiaceae & Xirrole & Root & Helminthiases & Decoction & Oral & 0.07 & 0.07 \\
\hline Celosia sp. 7390 & Amaranthaceae & Vela valheka & Fruit & Furuncle & $\begin{array}{l}\text { Burn into powder, } \\
\text { add Ricinus } \\
\text { communis seed oil }\end{array}$ & Topical & 0.07 & 0.07 \\
\hline $\begin{array}{l}\text { Combretum molle } \\
\text { R.Br. ex G.Don. } \\
\text { CM1520 }\end{array}$ & Combretaceae & $\begin{array}{l}\text { Xicucutse, } \\
\text { Xiwondzwana }\end{array}$ & Root & $\begin{array}{l}\text { Diarrhea, dysentery, } \\
\text { helminthiases, UTI, wounds }\end{array}$ & Decoction & Oral & 0.20 & 0.33 \\
\hline Croton sp. 3724 & Euphorbiaceae & Tchequelanhama & Root & Epilepsy & Decoction & Oral & 0.07 & 0.07 \\
\hline \multirow[t]{2}{*}{$\begin{array}{l}\text { Cucumis africanus } \\
\text { L.f. MxiqDFB11 }\end{array}$} & \multirow[t]{2}{*}{ Cucurbitaceae } & \multirow[t]{2}{*}{ Chiracarane } & \multirow[t]{2}{*}{ Root } & \multirow[t]{2}{*}{$\begin{array}{l}\text { Helminthiases, } \\
\text { Schistosomiasis }\end{array}$} & $\begin{array}{l}\text { Decoction or Cold } \\
\text { infusion for } 15 \text { min }\end{array}$ & Oral & \multirow[t]{2}{*}{0.20} & \multirow[t]{2}{*}{0.13} \\
\hline & & & & & Cold infusion & Rectal & & \\
\hline $\begin{array}{l}\text { Dichrostachys } \\
\text { cinerea (L.) Wight } \\
\text { \& Arn. } 4166\end{array}$ & Fabaceae & Tsenga & Fruit & Wounds & Burn into powder & Topical & 0.07 & 0.07 \\
\hline $\begin{array}{l}\text { Elephantorrhiza } \\
\text { elephantina } \\
\text { (Burch.) Skeels } \\
\text { MxipDFB01 }\end{array}$ & Fabaceae & Xivurai & Root & $\begin{array}{l}\text { Helminthiases, diarrhea } \\
\text { (bloody or not), cough, } \\
\text { tuberculosis, dysentery, } \\
\text { haemorrhoids }\end{array}$ & Decoction & Oral & 0.80 & 0.40 \\
\hline $\begin{array}{l}\text { Garcinia } \\
\text { livingstonei T. } \\
\text { Anderson } \\
\text { MxiqDFB20 }\end{array}$ & Clusiaceae & Bimbe, Mahimbe & $\begin{array}{l}\text { Root } \\
\text { and } \\
\text { stem }\end{array}$ & $\begin{array}{l}\text { Helminthiases, diarrhea, } \\
\text { cough, dysentery }\end{array}$ & Decoction & Oral & 0.47 & 0.27 \\
\hline \multirow{2}{*}{$\begin{array}{l}\text { Gladiolus sp. } \\
\text { PSM1139 }\end{array}$} & \multirow[t]{2}{*}{ Iridaceae } & \multirow[t]{2}{*}{ Halahingwa } & \multirow{2}{*}{$\begin{array}{l}\text { Bulb } \\
\text { and } \\
\text { root }\end{array}$} & Diarrhea, dysentery & Decoction & Oral & \multirow[t]{2}{*}{0.20} & \multirow[t]{2}{*}{0.27} \\
\hline & & & & $\begin{array}{l}\text { Helminthiases, } \\
\text { Schistosomiasis }\end{array}$ & Cold infusion & Rectal & & \\
\hline
\end{tabular}


Table 2 Medicinal plants sold for treatment of bacterial and parasitic diseases in Xipamanine, Xiquelene and Mazambane markets, Mozambique (Continued)

\begin{tabular}{|c|c|c|c|c|c|c|c|c|}
\hline $\begin{array}{l}\text { Scientific name / } \\
\text { voucher no }\end{array}$ & Family & Local name & $\begin{array}{l}\text { Parts } \\
\text { used }\end{array}$ & Ailment treated & Preparation & Administration & RFC & UV \\
\hline $\begin{array}{l}\text { Grewia sulcata } \\
\text { Mast. } 8270\end{array}$ & Tiliaceae & Chiuane & Root & Cough & Decoction & Oral & 0.07 & 0.07 \\
\hline $\begin{array}{l}\text { Gymnanthemum } \\
\text { coloratum (Willd.) } \\
\text { H.Rb. \& B. Kahn } \\
\text { MxipDFB05 }\end{array}$ & Asteraceae & Nhathelo, Palhakufa & Root & Helminthiases & Decoction & Oral & 0.07 & 0.07 \\
\hline $\begin{array}{l}\text { Gymnosporia } \\
\text { heterophylla (Eckl. } \\
\text { \& Zeyh.) Loes } \\
\text { MxiqDFB03 }\end{array}$ & Celastraceae & Xihlangua & Root & Diarrhea, dysentery & Decoction & Oral & 0.27 & 0.13 \\
\hline $\begin{array}{l}\text { Heinsia crinita } \\
\text { (Afzel.) G. Taylor } \\
\text { KGD9652 }\end{array}$ & Rubiaceae & Xissindze & Root & Helminthiases & Decoction & Oral & 0.07 & 0.07 \\
\hline \multirow{2}{*}{$\begin{array}{l}\text { Hugonia orientalis } \\
\text { Engl. PC2349 }\end{array}$} & \multirow[t]{2}{*}{ Linaceae } & \multirow[t]{2}{*}{ Congulutamute } & \multirow[t]{2}{*}{ Root } & Diarrhea & Decoction & Oral & \multirow[t]{2}{*}{0.13} & \multirow[t]{2}{*}{0.13} \\
\hline & & & & Wounds & Cold infusion & Topical - wash & & \\
\hline $\begin{array}{l}\text { Hydnora } \\
\text { abyssinica A.Br. } \\
\text { MxipDFB14 }\end{array}$ & Aristolochiaceae & Mavumbule & Rhizome & $\begin{array}{l}\text { UTI, helminthiases, internal } \\
\text { wounds }\end{array}$ & Decoction & Oral & 0.20 & 0.20 \\
\hline $\begin{array}{l}\text { Hypoxis } \\
\text { hemerocallidea } \\
\text { Fisch., C.A.Mey. \& } \\
\text { Avé-Lall. } \\
\text { MxiqDFB4 }\end{array}$ & Hypoxidaceae & Batata africana & Corm & $\begin{array}{l}\text { Helminthiases, diarrhea } \\
\text { (bloody or not), dysentery, } \\
\text { wounds (internal or } \\
\text { external), UTI, } \\
\text { haemorrhoids }\end{array}$ & $\begin{array}{l}\text { Decoction or Cold } \\
\text { infusion }\end{array}$ & Oral & 0.53 & 0.40 \\
\hline $\begin{array}{l}\text { Kedrostis sp. } \\
\text { MxiqDFB06 }\end{array}$ & Cucurbitaceae & $\begin{array}{l}\text { Dema amarelo, } \\
\text { Dema }\end{array}$ & Root & $\begin{array}{l}\text { Diarrhea, helminthiases, } \\
\text { UTI, wounds }\end{array}$ & $\begin{array}{l}\text { Decoction or Cold } \\
\text { infusion }\end{array}$ & Oral & 0.27 & 0.27 \\
\hline $\begin{array}{l}\text { Kigelia africana } \\
\text { (Lam.) Benth. } 9781\end{array}$ & Bignoniaceae & Mpfungura & Fruit & Deep wounds & $\begin{array}{l}\text { Burn into powder, } \\
\text { add R. communis } \\
\text { seed. Cover the } \\
\text { wound with honey } \\
\text { first. }\end{array}$ & Topical & 0.07 & 0.07 \\
\hline $\begin{array}{l}\text { Maclura africana } \\
\text { (Bureau) Corner } \\
\text { GD617 }\end{array}$ & Moraceae & Npumbulu & Root & Helminthiases & Decoction & Oral & 0.07 & 0.07 \\
\hline $\begin{array}{l}\text { Maerua juncea Pax } \\
\text { PM954 }\end{array}$ & Capparaceae & Chipinga & Root & Helminthiases & Decoction & Oral & 0.13 & 0.07 \\
\hline $\begin{array}{l}\text { Mangifera indica L. } \\
7736\end{array}$ & Anacardiaceae & Mangueira & Root & Helminthiases & Decoction & Oral & 0.07 & 0.07 \\
\hline $\begin{array}{l}\text { Margaritaria } \\
\text { discoidea (Baill.) } \\
\text { G.L.Webster } \\
\text { MxiqDFB01 }\end{array}$ & Phyllanthaceae & Xindikwe, Sinderane & Root & UTI, tuberculosis, cough & Decoction & Oral & 0.20 & 0.20 \\
\hline $\begin{array}{l}\text { Mucuna coriacea } \\
\text { Baker MxiqDFB18 }\end{array}$ & Fabaceae & Fethla & Root & $\begin{array}{l}\text { Helminthiases, internal } \\
\text { wounds }\end{array}$ & Decoction & Oral & 0.13 & 0.13 \\
\hline $\begin{array}{l}\text { Ochna natalitia } \\
\text { (Meisn.) Walp. } \\
\text { MxiqDFB19 }\end{array}$ & Ochnaceae & Mathlanganisso & Root & Helminthiases, tuberculosis & Decoction & Oral & 0.13 & 0.13 \\
\hline $\begin{array}{l}\text { Opilia amentacea } \\
\text { Roxb. GKD1159 }\end{array}$ & Opiliaceae & Magunthlo & Root & Helminthiases & Decoction & Oral & 0.07 & 0.07 \\
\hline $\begin{array}{l}\text { Opuntia ficus- } \\
\text { indica (L.) Mill. 10, } \\
485\end{array}$ & Cactaceae & Xihaca & Stem & Cough & $\begin{array}{l}\text { Cut and make syrup } \\
\text { with honey }\end{array}$ & Oral & 0.07 & 0.07 \\
\hline $\begin{array}{l}\text { Ozoroa obovata } \\
\text { (Oliv.) R. Fern. \& A. } \\
\text { Fern. } 7771\end{array}$ & Anacardiaceae & Chinungo & Root & Wounds & Cold infusion & Topical - wash & 0.07 & 0.07 \\
\hline Phyllanthus & Phyllanthaceae & Tetenha & Root & Diarrhea & Decoction & Oral & 0.07 & 0.07 \\
\hline
\end{tabular}


Table 2 Medicinal plants sold for treatment of bacterial and parasitic diseases in Xipamanine, Xiquelene and Mazambane markets, Mozambique (Continued)

\begin{tabular}{|c|c|c|c|c|c|c|c|c|}
\hline $\begin{array}{l}\text { Scientific name / } \\
\text { voucher no }\end{array}$ & Family & Local name & $\begin{array}{l}\text { Parts } \\
\text { used }\end{array}$ & Ailment treated & Preparation & Administration & RFC & UV \\
\hline \multicolumn{9}{|l|}{$\begin{array}{l}\text { reticulatus Poir. } \\
8395\end{array}$} \\
\hline $\begin{array}{l}\text { Secamone } \\
\text { punctulata Decne. } \\
9588\end{array}$ & Apocynaceae & Ximufane & Root & Diarrhea & Decoction & Oral & 0.07 & 0.07 \\
\hline $\begin{array}{l}\text { Senna occidentalis } \\
\text { (L.) Link } 3269\end{array}$ & Fabaceae & $\begin{array}{l}\text { Nhokane, Ndlha } \\
\text { nhoka, Ndlha } \\
\text { nhokane, Nhokane } \\
\text { tsongo, Nhokane } \\
\text { uculo }\end{array}$ & Root & Helminthiases, diarrhea & Decoction & Oral & 0.40 & 0.13 \\
\hline $\begin{array}{l}\text { Senna petersiana } \\
\text { (Bolle) Lock } 4301\end{array}$ & Fabaceae & $\begin{array}{l}\text { Nembe-nembe } \\
\text { uculo }\end{array}$ & Root & Helminthiases, epilepsy & Decoction & Oral & 0.13 & 0.13 \\
\hline $\begin{array}{l}\text { Spirostachys } \\
\text { africana Sond. } \\
\text { MmazDFB03 }\end{array}$ & Euphorbiaceae & $\begin{array}{l}\text { Mubhandwa, } \\
\text { Chilangamalho }\end{array}$ & $\begin{array}{l}\text { Root } \\
\text { and } \\
\text { stem }\end{array}$ & $\begin{array}{l}\text { Diarrhea, epilepsy, } \\
\text { dysentery }\end{array}$ & Decoction & Oral & 0.33 & 0.20 \\
\hline $\begin{array}{l}\text { Strychnos } \\
\text { decussata (Pappe) } \\
\text { Gilg } 3050\end{array}$ & Loganiaceae & Xinkwakwani & Root & Helminthiases & Decoction & Oral & 0.07 & 0.07 \\
\hline \multirow{2}{*}{$\begin{array}{l}\text { Strychnos } \\
\text { henningsii Gilg } \\
1740\end{array}$} & \multirow[t]{2}{*}{ Loganiaceae } & \multirow[t]{2}{*}{ Manono } & Root & Abdominal pain & \multirow{2}{*}{$\begin{array}{l}\text { Cold infusion or } \\
\text { decoction }\end{array}$} & \multirow[t]{2}{*}{ Oral } & \multirow[t]{2}{*}{0.13} & \multirow[t]{2}{*}{0.13} \\
\hline & & & $\begin{array}{l}\text { Root } \\
\text { bark }\end{array}$ & Diarrhea with pain & & & & \\
\hline $\begin{array}{l}\text { Strychnos spinosa } \\
\text { Lam. } 1738\end{array}$ & Loganiaceae & Massala & Root & Helminthiases & Decoction & Oral & 0.13 & 0.07 \\
\hline $\begin{array}{l}\text { Tabernaemontana } \\
\text { elegans Stapf } 8923\end{array}$ & Apocynaceae & Ncahlu & Root & Diarrhea, wounds, UTI & Decoction & Oral & 0.20 & 0.20 \\
\hline \multirow[t]{2}{*}{$\begin{array}{l}\text { Terminalia sericea } \\
\text { Burch. ex DC. } \\
\text { MxipDFB02 }\end{array}$} & \multirow[t]{2}{*}{ Combretaceae } & \multirow[t]{2}{*}{ Conola } & $\begin{array}{l}\text { Root, } \\
\text { leaves }\end{array}$ & $\begin{array}{l}\text { Diarrhea (bloody or not), } \\
\text { dysentery, helminthiases, } \\
\text { haemorrhoids }\end{array}$ & Decoction & Oral & \multirow[t]{2}{*}{0.87} & \multirow[t]{2}{*}{0.33} \\
\hline & & & $\begin{array}{l}\text { Root } \\
\text { bark }\end{array}$ & Wounds & $\begin{array}{l}\text { Dry and grind into } \\
\text { power }\end{array}$ & Topical & & \\
\hline $\begin{array}{l}\text { Tiliacora funifera } \\
\text { (Miers) Oliv. } \\
\text { MmazDFB01 }\end{array}$ & Menispermaceae & Xiwizila & Root & Helminthiases, diarrhea & Decoction & Oral & 0.60 & 0.13 \\
\hline $\begin{array}{l}\text { Warburgia } \\
\text { salutaris (G.Bertol.) } \\
\text { Chiov. 10,615 }\end{array}$ & Canellaceae & Xibaha & $\begin{array}{l}\text { Root } \\
\text { bark }\end{array}$ & Mouth ulcers, cough & Decoction & Oral & 0.13 & 0.13 \\
\hline $\begin{array}{l}\text { Zanthoxylum } \\
\text { capense (Thunb.) } \\
\text { Harv. K8014 }\end{array}$ & Rutaceae & Manunguane & Root & UTI, helminthiases & Decoction & Oral & 0.13 & 0.13 \\
\hline Unidentified & - & Xipenele & Root & Helminthiases & Decoction & Oral & 0.07 & 0.07 \\
\hline Unidentified & - & Wutambuti & Root & UTI & Decoction & Oral & 0.07 & 0.07 \\
\hline Unidentified & - & Tsatsalane & Leaf & Helminthiases & Decoction & Oral & 0.07 & 0.07 \\
\hline Unidentified & - & Towane & Root & Diarrhea & Decoction & Oral & 0.07 & 0.07 \\
\hline Unidentified & - & Tchongo & Root & Helminthiases & Decoction & Oral & 0.07 & 0.07 \\
\hline Unidentified & - & Nicungo & Root & Wounds & Grind into powder & Topical & 0.07 & 0.07 \\
\hline Unidentified & - & Nhanho & Root & Haemorrhoids & $\begin{array}{l}\text { Decoction or burn } \\
\text { into powder }\end{array}$ & Rectal & 0.07 & 0.07 \\
\hline Unidentified & - & NhacutsIwani & Root & Diarrhea & Decoction & Oral & 0.07 & 0.07 \\
\hline Unidentified & - & Nandzelate & Leaf & UTI & Decoction & Oral & 0.07 & 0.07 \\
\hline Unidentified & - & Massoliza & Root & Helminthiases & Decoction & Oral & 0.07 & 0.07 \\
\hline Unidentified & - & Magazine & Root & Diarrhea & Decoction & Oral & 0.07 & 0.07 \\
\hline Unidentified & - & Magaranhaca & Root & Epilepsy & Decoction & Oral & 0.07 & 0.07 \\
\hline
\end{tabular}


Table 2 Medicinal plants sold for treatment of bacterial and parasitic diseases in Xipamanine, Xiquelene and Mazambane markets, Mozambique (Continued)

\begin{tabular}{|c|c|c|c|c|c|c|c|c|}
\hline $\begin{array}{l}\text { Scientific name / } \\
\text { voucher no }\end{array}$ & Family & Local name & $\begin{array}{l}\text { Parts } \\
\text { used }\end{array}$ & Ailment treated & Preparation & Administration & RFC & UV \\
\hline Unidentified & - & Lilhatana & Root & Helminthiases & Decoction & Oral & 0.07 & 0.07 \\
\hline \multirow[t]{2}{*}{ Unidentified } & - & Chepa & Root & Wounds & Cold infusion & $\begin{array}{l}\text { Topical - } \\
\text { wash }\end{array}$ & 0.13 & 0.13 \\
\hline & & & & Cough & Decoction & Oral & & \\
\hline
\end{tabular}

aTuberculosis of the respiratory system

are mixed for treatment of furuncles. Burned Solanum tuberosum (known as "batata reno") tuber is also added to $R$. communis seed oil for the same purpose. Sclerocarya birrea ("canhueiro") and Anacardium occidentale ("cajueiro") root barks are decocted to treat cough. Cold infusion of Allium sativum (known as "xinhalane" or "alho") bulb is taken to treat helminthiases.

Some plants are also used for patient recovery after medicinal treatment. For example, Elephantorrhiza elephantina root and Ximenia americana (locally known as "tunduluka") root bark are used to treat weakness that results from helminthiases infection. A cold infusion is made and the filtrate is used to make corn porridge that is ingested for one week.

\section{Discussion}

A large diversity of plants was recorded as sold for treatment of bacterial and parasitic diseases. It was also found that there is still a high trade of plants in Maputo markets, and a high demand by the population. Most of the plants cited in this study were documented in one previous study done in 2004, although some vernacular names differ [6].
The vendors did not provide detailed information about how they collect or obtain the plants, i.e. if they collect themselves or acquired by other means. However, many explained that the plants were collected in many districts from Maputo city and province, namely: KaTembe, Boane, Manhiça, Moamba, Marracuene and Matutuine, and in Gaza and Inhambane, the nearby provinces also located in southern Mozambique. According to one of the vendors, $W$. salutaris was the only species collected cross border in South Africa. Other vendors affirmed that it was collected from Moamba district in Maputo province and at Inhambane province. This information is worrisome because this plant is endangered according to the IUCN Red List of Threatened Species 1998 [22].

It was important to record where the plants are harvested, mainly in Maputo province. The majority of districts in the province are rapidly getting urbanized because of house constructions. Therefore, there is an increased risk of deforestation and this can threaten the availability of the medicinal plants. Moreover, it might be difficult for vendors to obtain the plants in the future.

All of the most cited plants in this study had a high FL for diarrhea. The higher value of FL was found for

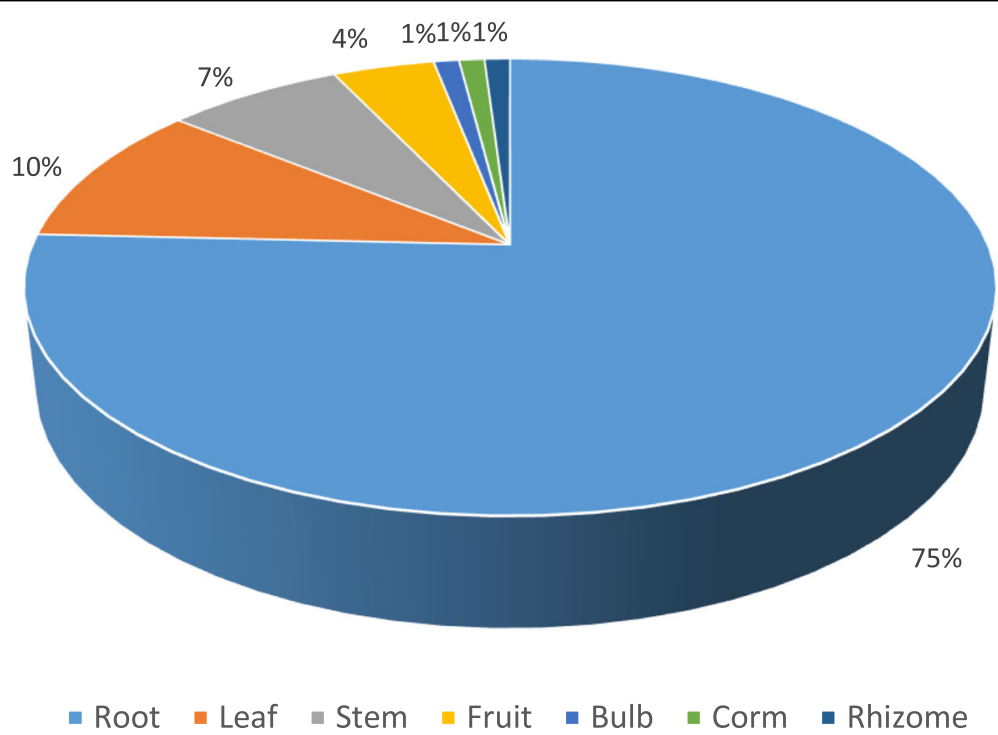

Fig. 3 Frequency of sold plant parts 
Table 3 Most cited diseases by the informants by medical category and disease

\begin{tabular}{|c|c|c|c|}
\hline Medical category ${ }^{a}$ & Disease & $\mathrm{n}$ & $\%$ \\
\hline \multirow[t]{4}{*}{ Certain infectious or parasitic diseases } & Helminthiases & 33 & $28 \%$ \\
\hline & Dysentery & 8 & $7 \%$ \\
\hline & Tuberculosis ${ }^{b}$ & 5 & $4 \%$ \\
\hline & Schistosomiasis & 2 & $2 \%$ \\
\hline \multirow[t]{3}{*}{ Diseases of the digestive system } & Diarrhea & 21 & $18 \%$ \\
\hline & Haemorrhoids & 5 & $4 \%$ \\
\hline & Abdominal Pain & 1 & $1 \%$ \\
\hline Injury, poisoning or certain other consequences of external causes & Wounds & 19 & $16 \%$ \\
\hline Symptoms or signs involving the respiratory system & Cough & 11 & $9 \%$ \\
\hline Diseases of the genitourinary system & UTI & 11 & $9 \%$ \\
\hline Diseases of the nervous system & Epilepsy & 4 & $3 \%$ \\
\hline
\end{tabular}

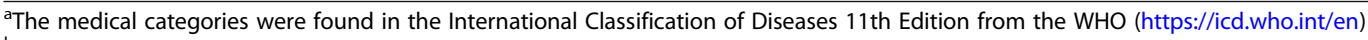

${ }^{\mathrm{b}}$ Tuberculosis of the respiratory system

Terminalia sericea (=0.91), followed by Elephantorrhiza elephantina, Hypoxis hemerocallidea and Tiliacora funifera with values of $0.88,0.75$ and 0.44 , respectively. All these plants are native from Africa and Mozambique.

T. sericea is widely used in the traditional medicine in this continent [23]. It was among the most traded medicinal plants in Mpumalanga Province, South Africa [24]. Among other medicinal uses reported in South Africa, roots were commonly used to treat diarrhea and infectious diseases [25]. Phytochemical studies were conducted and most of the active ingredients were isolated from the roots and stem bark [23].

Several in vitro studies were conducted for $T$. sericea [26-28]. For example works on intestinal infectioncausing bacteria showed that $T$. sericea is effective against various bacteria such as Micrococcus luteus, Enterobacter aerogenes, Streptoccocus pyogenes and Staphylococcus aureus [26, 27]. Other study has reported the activity of ethyl acetate root extract against Bacillus subtilis and Escherichia coli, with MICs of 0.3 and 1.5 $\mathrm{mg} / \mathrm{ml}$, respectively. These data shows that $T$. sericea is effective against various pathogenic microorganisms, including enteric pathogens [28].

Elephantorrhiza elephantina, was also cited in other South African studies for the treatment of diarrhea, helminthiases but results from this study revealed other diseases including cough, tuberculosis, dysentery and haemorrhoids. Active principles have already been isolated and biological activity against several pathogens has been studied, with positive results $[29,30]$.

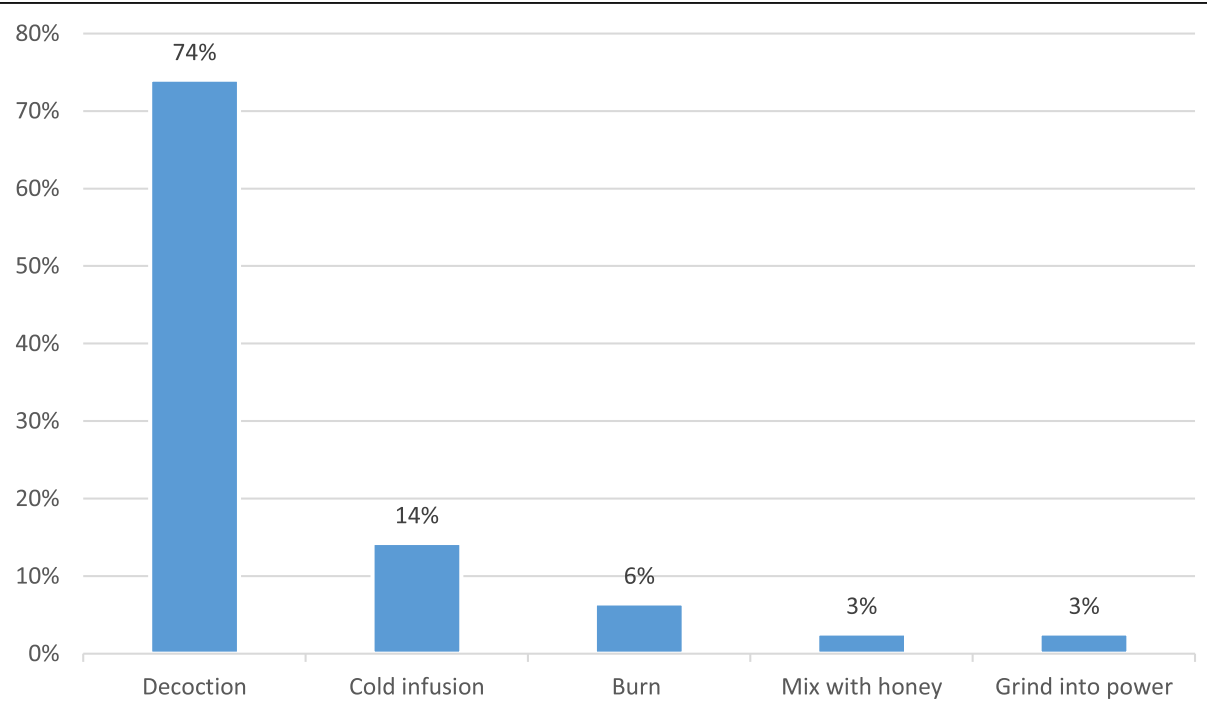

Fig. 4 Frequency of medicinal plant preparation methods 
Table $4 \mathrm{FL}$ values for the most cited medicinal plants

\begin{tabular}{lllll}
\hline Plant & Disease & Np & Ns & FL \\
\hline Terminalia sericea Burch. ex DC. & Diarrhea & 10 & 11 & 0.91 \\
& Helminthiases & 2 & 11 & 0.18 \\
& Haemorrhoids & 1 & 11 & 0.09 \\
& Wounds & 1 & 11 & 0.09 \\
& Dysentery & 1 & 11 & 0.09 \\
Elephantorrhiza elephantina (Burch.) Skeels & Diarrhea & 7 & 8 & 0.88 \\
& Cough & 3 & 8 & 0.38 \\
& Helminthiases & 1 & 8 & 0.25 \\
& Tuberculosis & 1 & 8 & 0.13 \\
& Dysentery & 1 & 8 & 0.13 \\
& Haemorrhoids & 1 & 8 & 0.13 \\
Tiliacora funifera (Miers) Oliv. & Helminthiases & 5 & 9 & 0.56 \\
Hypoxis hemerocallidea Fisch. \& C.A. Mey & Diarrhea & 4 & 9 & 0.44 \\
& Diarrhea & 6 & 8 & 0.75 \\
& Wounds & 2 & 8 & 0.25 \\
& Helminthiases & 1 & 8 & 0.13 \\
& Dysentery & 1 & 8 & 0.13 \\
& UTl & 1 & 8 & 0.13 \\
& Haemorrhoids & 1 & 8 & 0.13 \\
\hline & & &
\end{tabular}

Hypoxis hemerocallidea, also known as a miracle plant because it has various therapeutic uses has been used in traditional African medicine for many years. The plant has been extensively studied at the laboratory level for a variety of purposes beyond those reported in this research [31]. The plant had higher FL for diarrhea. It has also been cited for the treatment of other diseases such as wounds, helminthiases dysentery, Urinary Tract Infection (UTI) and haemorrhoids. The antibacterial activity of corm and leaves extracts was positive against enteric bacteria and other organisms [32].

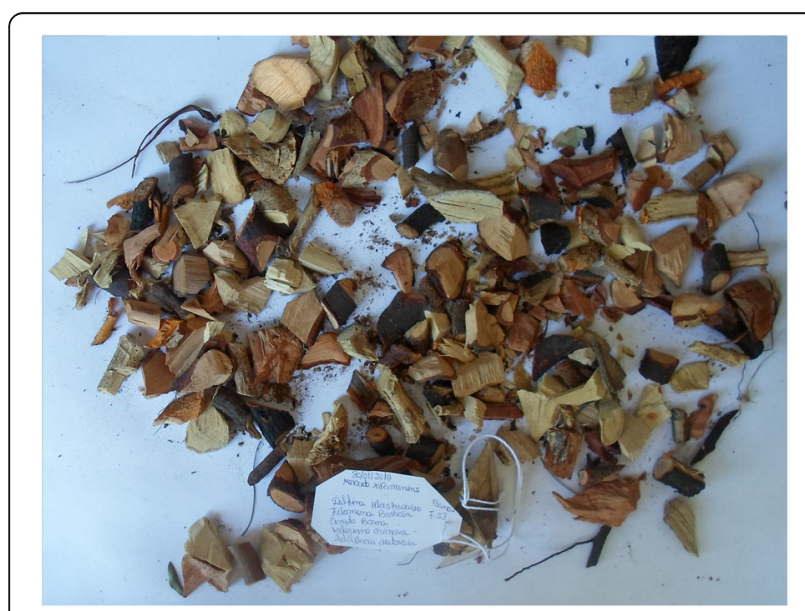

Fig. 5 Plants sold in Xipamanine market
Tiliacora funifera, cited for treatment of helminthiases and diarrhea, has anti-plasmodium activity due to alkaloids [33]. Ethnobotanical studies report that T. funifera root contributes to women's fertility and the leaves are used for treatment of facial skin problems [34]. The sap of the leaves is used in herbal remedies to prevent insanity in Congo, while in Ghana it is used to treat gastric fever, hernia and menstrual disorders [35]. This plant was also cited by many vendors in the previous study done in the study sites [6].

No recent laboratory studies were found for T. funifera and ethnobotanical studies do not often report the antidiarrheal and antihelmintic use of the plant. Thus, in vitro studies to validate medicinal use of this species are crucial. Nevertheless, studies reporting antimicrobial activity of other Tiliacora species have been found [36].

Curiously, one vendor said that T. funifera cures any disease and is more effective than African potato (Hypoxis hemerocallidea). This is important to note once $H$. hemerocallidea is consistently harvested and widely used to treat AIDS-related illnesses in the African continent. Attention should be paid to overexploitation of these plants in their natural habitat.

Hydnora abissinica, specie cited to treat UTI, helminthiases and internal wounds, was first recorded in southern Mozambique in a previous study [20]. It is well known by traders and traditional healers, therefore widely used within traditional medicine in southern Africa. This plant is rarely found by botanicals [20]. Phytochemical studies showed high tannin concentration in rhizomes which imparts a strong astringency and this may explain its efficacy in treating ailments of the digestive tract [21].

Fourteen plant materials could not be determined to family and species level in this study because these plant species were not available in the markets during the study period. It is more so important that in view of their ethnopharmacological importance these plant species deserve future attention for determination purpose.

Information on plant phytochemical studies and biological assays allows to know if their traditional use is validated. This information will be useful for defining subsequent studies of these plants.

\section{Conclusions}

Many plants are sold for the treatment of bacterial and parasitic diseases in the three main medicinal plants markets in Maputo city. Most of the plants are found in Maputo province forests. Access to plants in Maputo is becoming difficult due to increasing urbanization. Some endangered plants are still being sold, which is something to be aware and to develop mitigation strategies.

Fourteen plant materials could not be determined to family and species level. Neverthless these plant species deserve future attention for determination purpose. 
Medicinal plants are still commonly used and traded, even in large urban centers such as Maputo city. This data shows that the population still uses traditional medicine to treat their diseases.

This register of medicinal plants is very important once an erosion of knowledge is taking place. Some plants have already been studied in vitro for biological activity but other plants should be studied in order to evaluate their efficacy. In future new drug candidate molecules can be developed based on these plants against multidrug resistant strains.

\section{Abbreviations}

FL: Fidelity Level; RFC: Relative Frequency of Citation; UTI: Urinary Tract Infection; UV: Use Value

\section{Acknowledgments}

The authors would like to thank all of the vendors and the heads of the commission of the sellers of the markets. We are also grateful to our colleagues Mr. Ernesto Nacamo and Aurélio Bechel for the support in the scientific identification of the plants.

\section{Authors' contributions}

$\mathrm{FB}, \mathrm{VS}$ and $\mathrm{AC}$ performed study design. $\mathrm{FB}, \mathrm{DH}, \mathrm{EB}, \mathrm{VC}$ and $\mathrm{AM}$ carried out the fieldwork work. All authors reviewed literature, analyzed the data, prepared the manuscript, provided revisions, and approved the final manuscript.

\section{Funding}

This research did not receive any specific grant from funding agencies in the public, commercial, or not-for-profit sectors.

\section{Availability of data and materials}

All datasets used and/or analyzed during the current study are available from the corresponding author on reasonable request.

\section{Ethics approval and consent to participate}

The study was not submitted to a bioethics committee, but it was authorized by the Head of Department of Biological Sciences and by the Maputo city's council. Based on previous studies, we asked for oral consent that was obtained for all vendors before the interviews. No vendor was coerced into participating in the study.

\section{Consent for publication}

Not applicable.

\section{Competing interests}

The authors declare that they have no competing interests.

\section{Author details}

'Departamento de Ciências Biológicas, Faculdade de Ciências, Universidade Eduardo Mondlane, Avenida Julius Nyerere nr 3453, Campus Principal, Maputo, Mozambique. ${ }^{2}$ Departamento de Química, Faculdade de Ciências, Universidade Eduardo Mondlane, Avenida Julius Nyerere nr 3453, Campus Principal, Maputo, Mozambique. ${ }^{3}$ Direcção de Ciências Animais, Instituto de Investigação Agrária de Moçambique, Avenida de Moçambique Km 1.5, Maputo, Mozambique

Received: 14 October 2019 Accepted: 23 December 2019

Published online: 23 January 2020

\section{References}

1. World Health Organization (WHO). Traditional medicine strategy 2014-2023. 2013. https://www.who.int/iris/bitstream/10665/92455/1/9789241506090_ eng.pdf?ua=1.

2. Bruschi $P$, Mancini M, Mattioli E, Morganti M, Signorini MA. Traditional uses of plants in a rural community of Mozambique and possible links with
Miombo degradation and harvesting sustainability. J Ethnobiol Ethnomed. 2014;10:59.

3. Matavele J, Habib M. Ethnobotany in Cabo Delgado, Mozambique: use of medicinal plants. Environ Dev Sustain. 2000;2:227-34

4. Williams VL, Moshoeu TJ, Alexander GJ. Reptiles sold as traditional medicine in Xipamanine and Xiquelene markets (Maputo, Mozambique). S Afr J Sci. 2016;112(7/8):1-9.

5. Bandeira SO, Gaspar F, Pagula FP. African Ethnobotany and healthcare: emphasis on Mozambique. Pharm Biol. 2002;39(1):70-3.

6. Krog M, Falcão MP, Olsen CS. Medicinal plant markets and trade in Maputo, Mozambique. In: Forest \& Landscape Working Papers no. 16. KVL.Denmark: Danish centre for Forest, Landscape and Planning; 2006.

7. WHO (2019). World health statistics 2019: monitoring health for the SDGs, sustainable development goals. Geneva: World Health Organization; 2019. https://apps.who.int/iris/bitstream/handle/10665/324835/9789241565707eng.pdf.

8. Nhampossa T, Mandomando I, Acacio S, Quintó L, Vubil D, Ruiz J, et al. Diarrheal disease in rural Mozambique: burden, risk factors and etiology of diarrheal disease among children aged 0-59 months seeking Care at Health Facilities. PLoS One. 2015;10(5):e0119824.

9. Cerveja BZ, Tucuzo RM, Madureira AC, Nhacupe N, Langa IA, Buene T, et al. Prevalence of intestinal parasites among HIV infected and HIV uninfected patients treated at the $1^{\circ}$ De Maio health Centre in Maputo, Mozambique. EC Microbiol. 2017;9(6):231-40.

10. http://apps.who.int/neglected_diseases/ntddata/sth/sth.html. Accessed 3 December 2019.

11. INE (2019). Divulgação dos resultados preliminares IV RGPH 2017. Available at: http:/www.ine.gov.mz/operacoes-estatisticas/censos/censo-2007/censo-2017/ divulgacao-os-resultados-preliminares-iv-rgph-2017/view. Accessed 8 May 2019.

12. DSMSAS e CMM. Plano director 2015-2019 Município de Maputo. Versão Preliminar Maio 2015 100pp Maputo, Mozambique; 2015 Available at: https://wwwmedicusmundimozambiqueorg/files/2018/02/Plan_Director_ Maputopdf Accesses 11 December 2019.

13. International Society of Ethnobiology, 2006. International Society of Ethnobiology Code of Ethics (with 2008 additions) http://ethnobiology.net/ code-of-ethics/.

14. Decreto no 19/2007, de 9 de Agosto, que aprova o Regulamento sobre Acesso e Partilha de Benefícios Provenientes de Recursos Genéticos e Conhecimento Tradicional Associado.

15. de Koning J. Checklist of vernacular plant names in Mozambique. Wageningen Agricultural University papers. 1993:274.

16. Angiosperm Phylogeny Group. An update of the angiosperm phylogeny group classification for the orders and families of flowering plants: APG IV. Bot J Linn Soc. 2016;181(1):1-20.

17. Tardío J, Pardo-de-Santayana M. Cultural importance indices: a comparative analysis based on the useful wild plants of southern Cantabria (northern Spain). Econ Botany. 2008:62:24-39.

18. Prance GT, Balee W, Boom BM, Carneiro RL. Quantitative ethnobotany and the case for conservation in Amazonia. Conserv Biol. 1987;1:296-310.

19. Friedman J, Yaniv Z, Dafni A, Palewitch D. A preliminary classification of the healing potential of medicinal plants, based on a rational analysis of an ethnopharmacological field survey among Bedouins in the Negev desert. Israel J Ethnopharmacol. 1986;16:275-87.

20. Williams VL, Falcão MP, Wojtasik EM. Hydnora abyssinica: Ethnobotanical evidence for its occurrence in southern Mozambique. S Afr J Bot. 2011;77: 474-8.

21. Yagi S, Chrétien F, Duval RE, Fontanay S, Maldini M, Piacente S, et al. Antibacterial activity, cytotoxicity and chemical constituents of Hydnora johannis roots. S Afr J Bot. 2012;78:228-34.

22. Hilton-Taylor C, Scott-Shaw R, Burrows J, Hahn N. Warburgia salutaris. The IUCN Red List of Threatened Species 1998: e.T30364A9541142. http://dx.doi. org/https://doi.org/10.2305/IUCN.UK.1998.RLTS.T30364A9541142.en. Accessed 16 Sep 2019

23. Mongalo NI, McGaw LJ., Segapelo TV, Finnie JF, Van Staden J. Ethnobotany, phytochemistry, toxicology and pharmacological properties of Terminalia sericea Burch. Ex DC. (Combretaceae) - a review. J Ethnopharmacol, 2016 194, 789-802. https://doi.org/https://doi.org/10.1016/j.jep.2016.10.072.

24. Dold AP Cocks ML. The trade in medicinal plants in the eastern Cape Province. South Africa S Afr J Sci. 2002;98(11):589-97.

25. Coates-Palgrave K. Trees of Southern Africa. 5th ed. Cape Town, Johannesburg, RSA: Struik Publishers; 1988. p. 34, 684-5. 
26. Fyhrquist P, Mwasumbi L, Haeggström CA, Vuorela H, Hiltunen R, Vuorela P. Ethnobotanical and antimicrobial investigation on some species of Terminalia and Combretum (Combretaceae) growing in Tanzania. J Ethnopharmacol. 2002;79:169-77.

27. Steenkamp V, Mathivha E, Gouws MC, Van Rensburg CE. Studies on antibacterial, antioxidant and fibroblast stimulation of wound healing remedies from South Africa. J Ethnopharmacol. 2004;95:353-7.

28. van Vuuren SF, Naidoo D. An antimicrobial investigation of plants used traditionally in southern Africa to treat sexually transmitted infections. J Ethnopharmacol. 2010;130:552-8.

29. Mathabe MC, Nikovola RV, Lall N, Nyazema NZ. Antibacterial activities of medicinal plants used for the treatment of diarrhoea in Limpopo Province. South Africa J Ethnopharmacol. 2006;105(1-2):286-29.

30. Maroyi A. Elephantorrhiza elephantina: traditional uses, Phytochemistry, and pharmacology of an important Medicinal plant species in southern Africa. Evid Based Complement Alternat Med. 2017:1-18.

31. Owira PMO, Ojewole JAO. 'African potato' (Hypoxis hemerocallidea corm): a plant-medicine for modern and 21st century diseases of mankind? - a review. Phytother Res. 2009;23:147-52.

32. Katerere DR, Eloff JN (2008). Anti-bacterial and anti-oxidant activity ofHypoxis hemerocallidea (Hypoxidaceae): can leaves be substituted for corms as a conservation strategy? S Afr J bot. 2008; 74: 613-616.

33. Ayim JS, Dwuma Badu D, Fiagbe NY, Ateya AM, Slatkin DJ, Knapp JE, Schiff PL. Constituents of west African plants. XXI. Tiliafunimine, a new imino bisbenzylisoquinoline alkaloid from Tiliacora funifera. Lloydia. 1977;40(6): $561-5$.

34. De Wet H, Van Wyk B-E. An ethnobotanical survey of southern African Menispermaceae. S Afr J Bot. 2008;74:2-9.

35. Oyen LPA. Tiliacora funifera (Miers) Oliv. In: Brink, M., Achigan-Daco, E.G. (Eds.), Plant Resources of Tropical Africa 16. Fibres. PROTA Foundation, Wageningen, Netherlands/CTA, Wageningen, Netherlands; 2010.

36. Makinde EA, Ovatlarnporn C, Adekoya AE, Nwabor OF, Olatunji OJ. Antidiabetic, antioxidant and antimicrobial activity of the aerial part of Tiliacora triandra. S Afr J Bot. 2019;125:337-43.

\section{Publisher's Note}

Springer Nature remains neutral with regard to jurisdictional claims in published maps and institutional affiliations.

Ready to submit your research? Choose BMC and benefit from:

- fast, convenient online submission

- thorough peer review by experienced researchers in your field

- rapid publication on acceptance

- support for research data, including large and complex data types

- gold Open Access which fosters wider collaboration and increased citations

- maximum visibility for your research: over $100 \mathrm{M}$ website views per year

At BMC, research is always in progress.

Learn more biomedcentral.com/submissions 